\title{
Una revisión de literatura del modelo efectual usando ToS
}

\author{
A literature review of the \\ effective model using ToS
}

\begin{abstract}
Resumen
El emprendimiento a nivel mundial ha sido fuente de inspiración para quienes han tomado la decisión de desarrollar y manejar un negocio asumiendo los múltiples riesgos. Esto ha llevado a la implementación del modelo efectual, como una lógica de experiencia empresarial que los emprendedores principiantes pueden utilizar en la etapa inicial caracterizada por la incertidumbre. El objetivo principal es introducir a los lectores a los principales elementos de esta teoría, en los diálogos científicos actuales y los debates que se han generado en torno al tema. Este artículo se apoya en investigaciones previas realizadas en las diferentes ciencias sociales para proponer una visión cronológica y teórica del tema, a partir de una retrospectiva en la que se examinan estudios previos que permitan una mejor toma de decisiones por parte del emprendedor, a partir de un análisis de 297 artículos de investigación usando como herramienta el Árbol de la Ciencia (Tree of Science). Los resultados mostraron que hay una ciencia para el emprendimiento, una lógica común que se ha observado en empresarios expertos llamado modelo efectual, que promete un método a seguir para abordar los problemas, mientras se hacen crecer los negocios. Palabras clave: Modelo efectuar; emprendimiento; efectividad; causalidad; árbol de la ciencia
\end{abstract}

\section{Abstract}

Entrepreneurship worldwide has been a source of inspiration for those who have made the decision to develop and run a business while assuming multiple risks. This has led to the implementation of the effective model, as a business experience logic that beginning entrepreneurs can use in the initial stage characterized by uncertainty. The main objective is to introduce readers to the main elements of this theory, in the current scientific dialogues and the debates that have been generated around the subject. This article is based on previous research carried out in the different social sciences to propose a chronological and theoretical vision of the subject, based on a retrospective in which previous studies that allow better decisionmaking by the entrepreneur are examined, based on an analysis of 297 research articles using the Tree of Science as a tool. The results showed that there is a science to entrepreneurship, a common logic that has been observed in savvy entrepreneurs called the effective model, that promises a method to follow to address problems, while growing businesses.

Keywords: Effective model; entrepreneurship; effectiveness; causality; tree of science 
El emprendimiento como deseo de desarrollar y manejar un negocio, integrado con múltiples riesgos, ha generado en los últimos años la implementación de un modelo causal que no siempre ha tenido los mejores resultados por los emprendedores novatos. Por este motivo, aparece el modelo efectual como una lógica de experiencia empresarial al que los emprendedores principiantes puentes recurrir en la etapa inicial de incertidumbre, aumentando la probabilidad de éxito. Por ejemplo, empresas como Facebook y Google iniciaron sus operaciones basadas en el modelo efectual.

Facebook inició en la Universidad de Harvard con el ideal de conectar y compartir información entre la comunidad universitaria, sin utilizar un plan de negocios para buscar inversionistas. Por otro lado, Google comenzó en la Universidad de Stanford a partir de una tesis doctoral, desarrollando servicios en un servidor ubicado debajo del escritorio de sus creadores.

Estos dos ejemplos muestran empresas exitosas que fueron creadas a partir de los recursos que tenían sus fundadores, es decir, aplicando un modelo efectual. Por lo tanto, el modelo efectual es una propuesta reciente que, como lo refieren Sarasvathy \& Dew (2005a), "ha tenido una tasa anual de crecimiento del 14\%" (Figura 1). Este registro da cuenta de cómo producción científica anual con relación al modelo efectual ha aumentado considerablemente en los últimos siete años, evidenciando que a partir del año 2011 la producción científica aumentó en un doscientos por ciento.

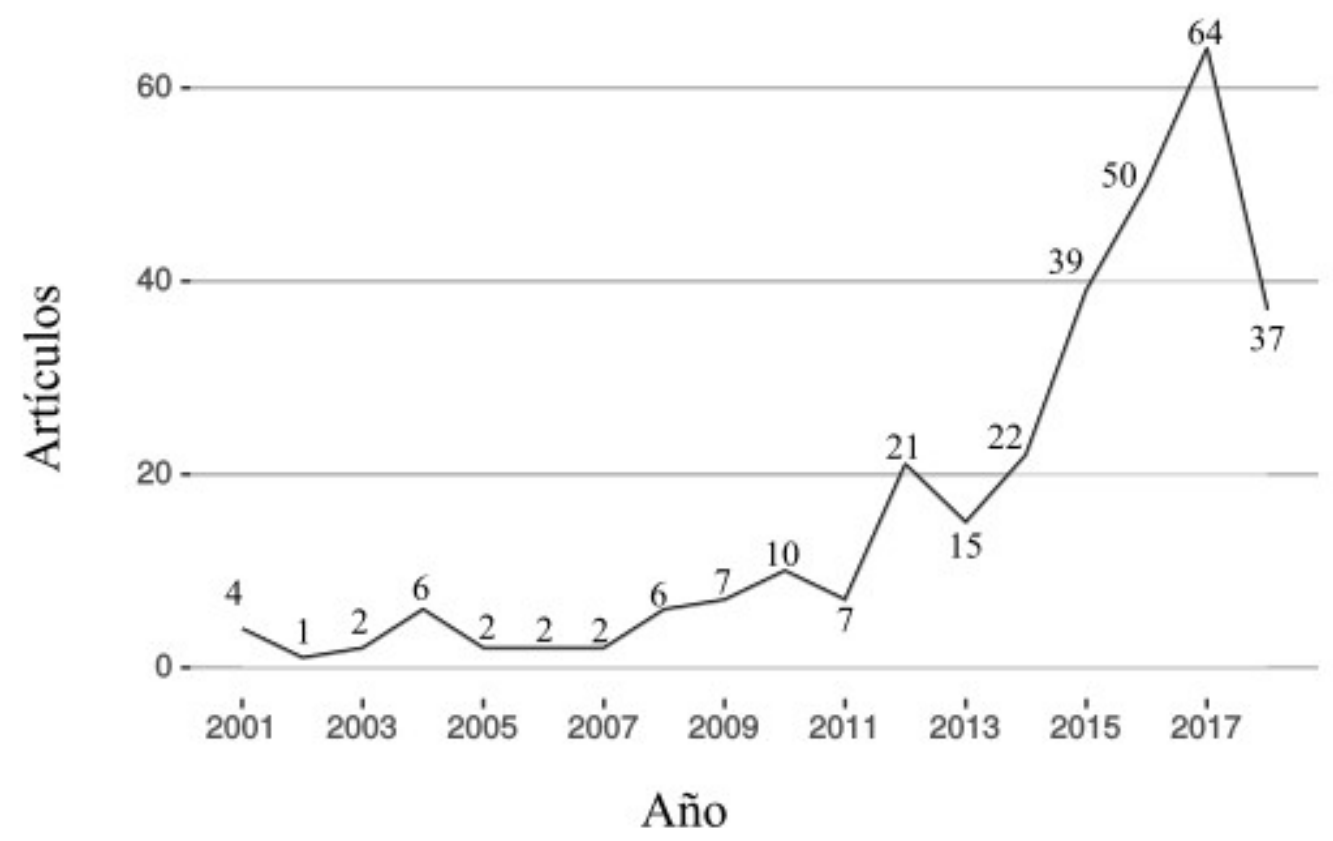

Figura 1. Producción Científica Anual sobre el modelo efectual. Fuente: Elaboración propia.

La producción científica sobre el tema afirma que, "no existe hasta la fecha un artículo de revisión que muestre la evolución cronológica de este concepto usando Tree of Science (ToS)" (Robledo, Osorio y López, 2014; Zuluaga et al., 2016). Sin embargo, existen aproximaciones a un análisis frente al tema como los adelantados por Perry, Chandler y Markova (2012), que realizaron una revisión de la literatura 
para resaltar la importancia de investigar el modelo efectual, pues pocos investigadores habían intentado probar empíricamente el modelo.

Adicionalmente, Matalamäki (2017), a través de su revisión, identificó las principales corrientes del modelo efectual: Desarrollo de producto e innovación, internacionalización, modelo efectual y causal de forma simultánea y la experiencia del emprendedor. Por último, Read, Song y Smit (2009) propusieron incorporar la posibilidad que la creación pueden ser las razones para algunos procesos emprendedores.

En este contexto, con el propósito de identificar las vertientes actuales del modelo efectual, se realiza el análisis de 297 artículos de investigación previamente revisados por los pares académicos. Para cumplir este objetivo, primero se realizó una consulta en Web of Science (Wos) del tema "modelo efectual", posteriormente se cargaron los resultados a la plataforma web Tree of Science, y finalmente, se identificaron los principales artículos de acuerdo con la posición en el árbol, los artículos clásicos en la raíz, los estructurales en el tronco y, por último, los artículos actuales en las hojas. La visualización en forma de árbol permite entender la evolución del concepto y los diferentes aportes a través del tiempo e identificar las diferentes perspectivas actuales del tema.

Desde el aspecto metodológico, el artículo explica de forma detallada el proceso de búsqueda e identificación de los principales artículos, los aportes de cada uno de estos de forma cronológica y las perspectivas identificadas a partir de las hojas.

\section{DisCUSIÓN TEÓRICA}

El modelo efectual, ha sido referenciado y enseñado dentro de las estrategias en las iniciativas emprendedoras en muchas escuelas de negocios, estando este basado en objetivos, la toma de decisiones o como un modelo de causalidad (Sarasvathy, 2001). El modelo efectual ha capturado la imaginación de los investigadores porque, identifica y cuestiona las suposiciones básicas de, cómo piensan y se comportan las personas al iniciar negocios, y ofrece una explicación alternativa a la causalidad que muchos creen que tiene validez. Actualmente existe mucha investigación en emprendimiento que genera la suposición fundamental de que se encuentran oportunidades. El modelo efectual, por ejemplo, posiciona al emprendedor como cocreador de oportunidades, junto con partes interesadas comprometidas (Read et al., 2009).

Según Sarasvathy (2001), se requiere la noción del modelo efectual y causalidad, que se argumenta en una lógica de predicción, es decir, es el efecto sobre la lógica del control lo que permitirá efectuar proposiciones para el trabajo empírico futuro, proponiendo llenar los vacíos construyendo un marco teórico del modelo efectual desde una perspectiva cronológica, mostrando los diferentes aportes y la evolución del modelo a través del tiempo.

Por otra parte, algunos autores entienden que la literatura sobre el espíritu empresarial internacional ha indicado que los empresarios constantemente aumentan sus actividades internacionales a lo largo de líneas de razonamiento inesperadas sin llegar a contar con un objetivo preciso, es decir, no planificado (Kalinic, Sarasvathy \& Forza, 2014). 
A su vez, los empresarios pueden continuar una lógica causal y pueden abordar sus decisiones sobre el principio de pérdida accesible en cambio de la maximización de los rendimientos esperados (Read et al., 2009). Por ende, aunque existan diversas investigaciones que dan fundamento de diversas técnicas y estrategias de emprendimiento, es evidente que, el modelo efectual busca posicionar al emprendedor como cocreador de oportunidades incluyendo a las partes interesadas, ejemplo del impacto que ha tenido este, es el metaanálisis de los artículos publicados en el Journal of Business Venturing, que resume los datos sobre 9897 nuevas empresas para conectar tres de los principios del modelo de manera positiva con el rendimiento de nuevas empresas, logrando medir con precisión la efectividad como un método general para extraer variables del trabajo previo que permita medir nuevos constructos.

Se destaca entonces como, muchas organizaciones ya establecidas, a partir de los nuevos segmentos de mercado y al mismo tiempo las demandas de los clientes han generado esfuerzos para emprender, abordando mercados adyacentes a las actividades propias. Eficacia y causalidad son dos lógicas que comprenden los efectos de los comportamientos empresariales en la puesta en marcha y en contextos adyacentes que se han mantenido sin explorar, "predominando el papel de las organizaciones industriales con relación a la efectividad de los comportamientos empresariales" (Futterer, Schmidt \& Heidenreich, 2018).

Por lo tanto, causalidad y modelo efectual son dos enfoques alternativos que utilizan los empresarios en el nuevo proceso de desarrollo de emprendimiento. Es importante mencionar que la causalidad es componente principal del enfoque planteado, al mismo tiempo, en condiciones de incertidumbre las circunstancias no permiten extraer inferencias estadísticas. No existe entonces, una forma confiable de calcular un rendimiento esperado para una acción realizada, por lo tanto, el emprendedor selecciona alternativas basadas en la pérdida de asequibilidad, utilizando la experimentación que permite ejercer control sobre el futuro, al realizar alianzas y compromisos con los proveedores, clientes y posibles competidores (Chandler, DeTienne, McKelvie \& Mumford, 2011).

\section{MÉTODOLOGíA}

Para la construcción del artículo, se utilizaron herramientas que permitieran obtener una respuesta más clara de la historia o evolución del modelo efectual a través de los años y sus diferentes perspectivas. La primera herramienta empleada fueron las bases de datos de la Universidad Nacional de Colombia en su sistema de red de bibliotecas llamada "Sinab", seleccionando en la base de datos indexada Web of Science aquellos artículos que tienen como temática el modelo efectual. Para esto se utilizó la siguiente ecuación de búsqueda: Topic $=($ Effectuation $)$ con un rango de fecha desde enero de 2000 hasta el 25 de septiembre de 2018, dando como resultado 297 artículos.

Una vez obtenidos estos resultados, se cargaron a la plataforma web Tree of Science (ToS). ToS ha sido aplicado en temas tan diversos que van desde la forma- 
ción ética en las organizaciones (Toro-Arias y Rodríguez, 2017), pasando por revisiones en temas ambientales (Cervantes, et al., 2018) y terminando en materiales sintéticos (Rodríguez-Sepúlveda \& Orrego-Alzate, 2016). ToS también posibilita la construcción y entendimiento práctico del estado del arte a partir de la búsqueda inicial de WoS.

El algoritmo de ToS se basa en la teoría de grafos, donde los artículos son representados como nodos y las citaciones entre ellos cómo links, de esta manera cada nodo representa una unidad de conocimiento ubicada dentro de la red. Los nodos más importantes son identificados a partir de su posición, y estos se determinan de acuerdo con los links que conectan a otros nodos. Por lo tanto, los artículos ubicados en la raíz son los más citados del tema del modelo efectual, los del tronco son los artículos que citan a la raíz y son citados por las hojas, siendo estas los artículos que citan tanto a la raíz como al tronco, de tal forma que, es posible visualizar la información científica en forma de árbol.

En la Figura 2 se puede observar los artículos seleccionados en forma de árbol, estructura a partir de la que se procedió a analizar y estudiar los artículos más importantes de cada parte del árbol y se seleccionaron cinco de cada área. Esta visualización permite tener una visión general de la historia, las bases y desarrollos actuales del modelo efectual, logrando una visión completa orientada a cumplir el objetivo principal de la investigación.

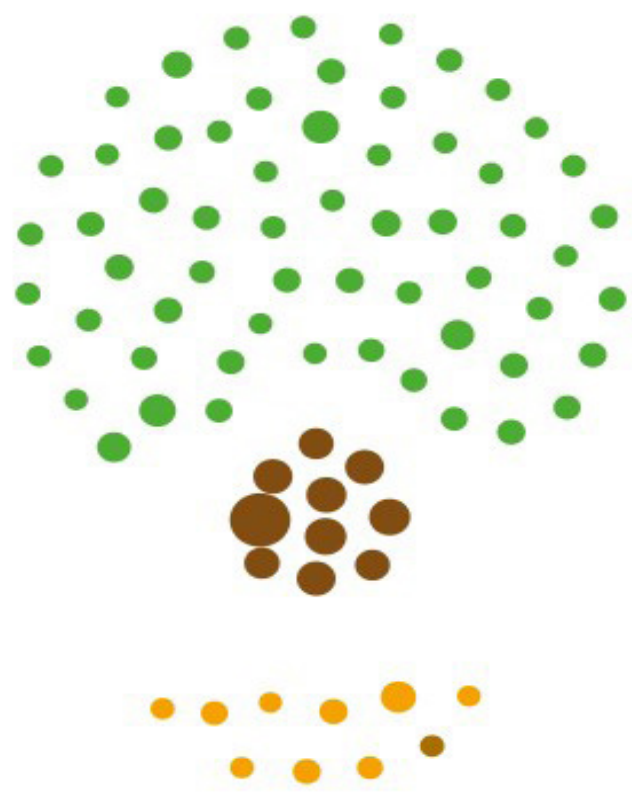

Figura 2. Artículos seleccionados como ejemplo del árbol para revisión bibliográfica. Fuente. Elaboración propia.
Hojas.

Shirokova et al. (2017),

Matalamaki (2017),

Mauer et al. (2018).

Tronco.

Arend, Sarooghi \& Burkemper (2015),

Wiltbank et al. (2006),

Sarasvathy et al. (2014).

Raiz.

Sarasvathy (2009),

Shane \& Venkataraman (2000),

Baker \& Nelson (2005).

Una vez seleccionadas las investigaciones, se realiza una descripción de los aspectos bibliométricos más importantes, tales como revistas e investigadores más representativos para el modelo efectual. Posteriormente, se analizaron los conceptos más importantes del modelo efectual con el fin de obtener una visión general del tema, 
clasificados de forma cronológica. A continuación, se describen los resultados de la investigación.

\section{Resultados}

\section{Revistas y artículos del modelo efectual}

Para realizar este análisis se utilizó el paquete de R Bibliometrix (Aria \& Cuccurullo, 2017) utilizando la semilla de 297 artículos (los resultados de WoS). Los 297 fueron publicados en 20 revistas diferentes, siendo Entrepreneurship y Regional Development, los más importantes, con un factor de impacto de 2.8 (Tabla 1). Todas las revistas están en Q1 lo que indica como el modelo efectual es un tema maduro y significativo dentro de la comunidad científica.

TABLA 1.

Las cuatro revistas científicas más representativas del modelo efectual.

\begin{tabular}{llll}
\hline \multicolumn{1}{c}{ Revistas científicas } & Artículos & Cuartil & F.I. \\
\hline Entrepreneurship and Regional Development. & 12 & Q1 & 2.8 \\
Entrepreneurship Theory and Practice. & 11 & $\mathrm{Q} 1$ & 4.9 \\
Journal of Business Venturing. & 11 & $\mathrm{Q} 1$ & 6.0 \\
International Entrepreneurship and Management Journal. & 10 & $\mathrm{Q} 1$ & 1.3 \\
Otros & 69 & - & - \\
\hline
\end{tabular}

Nota: Factor de Impacto del 2016.

Fuente. Elaboración propia.

Entre los autores más productivos se encuentran la doctora Sarasvathy (la creadora el pensamiento efectual) y el doctor Dew, entre otros (Tabla 2). Los cinco investigadores presentan índices h mayores a 12 lo que significa que el modelo efectual tiene figuras importantes en el mundo científico.

TABLA 2.

Los cinco investigadores más productivos del modelo efectual.

\begin{tabular}{lll}
\hline \multicolumn{1}{c}{ Revistas científicas } & Producción & Índice h \\
\hline Sarasvathy, S. & 16 & 45 \\
Dew, N. & 12 & 31 \\
Read, S. & 9 & 20 \\
Mauer, R. & 7 & 12 \\
Wiltbank, R. & 7 & $12^{*}$ \\
\hline
\end{tabular}

Nota: Índice h: De acuerdo con los datos de Google Scholar.

* A partir de los datos de Web of Science.

Fuente: Elaboración propia. 


\section{Tree of Science}

Los artículos ubicados en la raíz del "árbol de la ciencia", se pueden identificar como investigaciones que le dan soporte a la teoría del modelo efectual, son artículos que describen, de forma general, la importancia del emprendimiento corporativo y la noción del modelo efectual y causalidad. Por ejemplo, Sarasvathy (2001) plantea que, se deben realizar alianzas sin necesidad de llegar a un análisis competitivo y control de un futuro impredecible a través del modelo efectual, que puede ser utilizado como guía para el trabajo empírico. También Wiltbank, Dew, Read y Sarasvathy (2006) muestran que los empresarios expertos usan un efecto lógico que es transformador sin necesidad de recurrir a la predicción o creación de nuevos mercados y ambientes.

De otra parte, Read et al. (2009) abren el debate en torno al posicionamiento o construcción de las nuevas empresas, haciendo énfasis en los fundamentos teóricos en el núcleo del espíritu empresarial, la extracción de constructos relevantes de la literatura y el carácter distintivo de la iniciativa empresarial. Baker et al. (2005), indica que, los empresarios en entornos de escasos recursos, fueron capaces de prestar servicios únicos mediante la recombinación de elementos a mano para nuevos propósitos desafiando las definiciones institucionales y sus límites, así mismo, se encontró que el concepto de Lévi-Strauss de hacer bricolaje con lo que se tiene a mano, explica muchos de los comportamientos que observamos en pequeñas empresas que son capaz de crear a partir de la nada, mediante la explotación insumos físicos, sociales o institucionales que otras firmas rechazado o ignorado.

Algunos autores, dan importancia a las oportunidades empresariales independientes, que en la actualidad surgen de las percepciones, que están ahí, esperando a ser descubiertas o ser creadas por las acciones e iniciativas de los empresarios. Si bien siempre será posible visualizar y poner en marcha una oportunidad particular como ejemplo de un proceso de descubrimiento o creación, estas dos teorías tienen implicaciones importantes para la efectividad de una amplia variedad de acciones emprendedoras en diferentes contextos (Alvárez \& Barney, 2007).

El cuerpo principal de la investigación empresarial se basa en la toma de decisiones racionales, teniendo en cuenta que la mayoría de las oportunidades se descubren a través de un proceso de búsqueda con propósito, convirtiéndose en una ventaja competitiva para las empresas emergentes, determinada en gran medida por las competencias relacionadas con la búsqueda y explotación de oportunidades y los recursos controlados por la empresa. La mayoría de los investigadores del espíritu empresarial han supuesto que "los individuos participan en comportamientos racionales impulsados por metas cuando persiguen oportunidades" (Perry et al., 2012).

Es importante identificar cómo piensan y se comportan las personas al iniciar negocios. La efectividad parece ser particularmente apropiada para el emprendimiento porque, puede describir mejor como "en ausencia de mercados actuales para futuros bienes y servicios, estos llegan a existir" (Perry et al., 2012).

Para Read et al. (2009), la revisión meta-analítica de la ejecución y el rendimiento de la empresa es fundamental para establecer el modelo efectual en donde el emprendedor debe descubrir oportunidades y explotarlas, cuando se considera la creación de 
una nueva empresa desde esa perspectiva, es fácil ver la importancia de ideas tales como, la alerta emprendedora y la orientación empresarial, pero surge una perspectiva diferente llamada la efectividad, por consiguiente las oportunidades nacen cuando las crea un emprendedor y sus socios. En este contexto, una serie de ideas diferentes se vuelven importantes para comprender la creación de nuevas empresas.

El modelo efectual se está percibiendo como una teoría emergente de la iniciativa empresarial, proporcionando una explicación de por qué las personas terminan creando nuevas actividades comerciales, incluso cuando ese no era su objetivo inicial, tomando riesgos y sufriendo pérdidas, pero siempre conservando la capacidad de adaptación a los cambios provocados por el contexto, a esto se suma que, "los cambios en el entorno operativo también requieren que la empresa cambie y aprenda de nuevos métodos operativos para responder a situaciones cambiantes" (Matalamäki, 2017).

Dew, Read, Sarasvathy y Wiltbank (2009) investigaron las diferencias entre grupos de empresarios expertos y principiantes, encontrando diferencias relevantes que tienen implicaciones para la formación de empresarios potenciales, así mismo, para continuar las investigaciones futuras basadas en lograr trabajar en la cognición empresarial.

En cuanto a los artículos que se encuentran en el tronco, es decir, aquellos comienzan a dar forma a la teoría del "modelo efectual". Son artículos más específicos en el tema, que con el pasar de los años se han convertido en referentes. Entre los artículos que tenían mayor grado de intermediación, el primero que se encontró fue el trabajo de Wiltbank et al. (2006), que argumenta como, existen dos prescripciones que las empresas están llamadas a retomar en situaciones de incertidumbre, los enfoques de planificación y enfoques adaptativos.

Estos difieren principalmente en el rol apropiado de predicción en el proceso de decisión, siendo la predicción un tema central en la elaboración de estrategias, debido a la presunción de que lo que se puede predecir se puede controlar y como estos enfoques pueden afectar los costos y riesgos de estrategias firmes, así como los esfuerzos continuos de la empresa para innovar.

Así mismo, Sarasvathy, Kumar, York y Bhagavatula (2014) hacen referencia a las superposiciones, los desafíos y las posibilidades sobre el espíritu empresarial internacional que es investigado a través del lente teórico de la efectividad. Este se presenta como un enfoque eficaz al momento de resolver los conflictos centrales y lagunas de conocimiento involucrando nuevos métodos o nuevas formas organizativas, o una nueva unidad de análisis, haciendo hincapié en que no es necesario invertir más de lo que tenemos que perder para impulsar las fronteras actuales del emprendimiento.

Otros autores muestran como la nueva creación de mercadeo a través de la transformación del mercado, surgido desde procesos que involucran una nueva red de partes interesadas. La red se inicia a través de un compromiso efectivo que pone en movimiento los ciclos concurrentes de recursos en expansión y restricciones convergentes que resultan en el nuevo mercado (Sarasvathy \& Dew, 2005b).

En esta misma línea Chandler et al. (2011), exploran los procesos de causación y de ejecución a partir de un estudio de validación a través de medidas de causalidad y enfoques de efectividad para una nueva empresa, proponiendo que la ejecución se de- 
sarrolle mediante una construcción formativa, multidimensional con tres subdimensiones asociadas que son la experimentación, pérdida asequible y flexibilidad y una dimensión compartida con la causalidad constructo conocida como los compromisos previos, en el que la causalidad esta negativamente asociada con la incertidumbre mientras que la experimentación, una subdivisión de la efectividad, está positivamente correlacionado con la incertidumbre.

Desde otra mirada se habla de causalidad y efecto como un cambio teórico desde la inevitabilidad económica hacia la contingencia emprendedora, mientras la causalidad se basa en una lógica de predicción, efecto sobre la lógica del control, ilustrando la ejecución a través de ejemplos de negocios y experimentos de pensamiento realistas, examinando sus conexiones con teorías existentes y evidencia empírica, y ofrecen una lista de comprobables proposiciones para el trabajo empírico futuro. Los emprendedores exitosos han creado empresas, industrias e incluso las economías, haciendo coincidir los descendientes de imaginación humana con aspiraciones humanas, pues la creación de artefactos económicos exige inspiración y la prolongación de un esfuerzo cooperativo y competitivo (Sarasvathy, 2001).

Los productos innovadores son ampliamente reconocidos como una fuente importante de ventaja competitiva, sin embargo, muchas empresas tienen dificultades para encontrar enfoques eficientes y exitosos para diferentes tipos de proyectos, en particular aquellos que implican un alto nivel de innovación, en donde se desarrollan y diferencian de las estrategias convencionales basadas en la predicción de causalidad (Brettel, Mauer, Engelen \& Küpper, 2012).

En la toma de decisiones empresariales, los autores resaltan la presencia de la lógica efectiva versus la lógica predictiva, teniendo en cuenta las diferencias entre expertos y novatos, mostrando que los expertos emprendedores enmarcan las decisiones utilizando una lógica efectiva, identificando mercados potenciales, concentrados en la construcción de la empresa como un todo, prestando menos atención a la información predictiva, preocupándose más por hacer con los recursos disponibles, invirtiendo solo lo que podrían permitirse perder y enfatizando en la unión de redes de asociaciones, mientras los novatos usan un marco predictivo (Dew et al., 2009).

En la parte de las hojas, se ubican las diferentes perspectivas que se encontraron. El resultado del análisis arrojó que la efectuación proporciona una explicación de por qué las personas terminan creando nuevas actividades comerciales, incluso cuando ese no era su objetivo inicial cuando comenzaron sus operaciones, obteniendo como respuesta que ellos toman riesgos en la medida en que estén preparados para sufrir pérdidas y conservar la capacidad de adaptación a los cambios provocados por el medio ambiente, a la vez que, "persiguen nuevas oportunidades comerciales que surgen de los cambios pertinentes y aprender haciendo (Matalamäki, 2017).

Para analizar la comprensión de las condiciones de frontera y la aplicación exitosa de la búsqueda de procesos en el espacio del problema empresarial, se ha utilizado un agente basado en enfoque de simulación para investigar formalmente la influencia de la isotropía ambiental, imprevisibilidad y la ambigüedad de objetivos en dos procesos de búsqueda distintos, uno basado en la predicción y uno basado en control. En este sentido, Mauer, Wuebker, Schlüter \& Brettel (2018) investigaron el rendimiento de 
la ejecución como un ejemplo de la búsqueda y la causalidad no predictivas basadas en el control como un ejemplo de búsqueda basada en la predicción, cuyos resultados fortalecen la teoría, al revelar una relación más matizada entre el medio ambiente y la búsqueda empresarial más que el trabajo conceptual.

A su vez, se han realizado investigaciones sobre los efectos y los comportamientos empresariales en innovación a partir del modelo de negocio y rendimiento de las empresas. El Corporate Venturing establece el escenario para el comportamiento empresarial con el fin de crear negocios y un modelo de innovación, mediante un estudio que investiga el modelo efectual y la causalidad como "lógicas emprendedoras primarias, en donde la efectividad (causalidad) es más efectiva en un crecimiento industrial alto configuraciones" (Futterer et al., 2018).

Esta investigación presenta modelos basados en agentes, como un enfoque alternativo para avanzar en la investigación en emprendimiento. Se argumenta que los modelos basados en agentes permiten a los investigadores en emprendimiento encontrar mejores formas de generar resultados empresariales entendiendo una alternativa histórica y examinar un futuro plausible, abordando la brecha metodológica que existe en la investigación empresarial y resaltando la necesidad de una adopción más amplia del enfoque de simulación modelos basados en agentes, para estudiar el emprendimiento (Sunny \& Chandra, 2013).

Por su parte, Matalamäki, Vuorinen, Varamäki, \& Sorama (2017) han determinado que el crecimiento empresarial en empresas establecidas a través de los roles de eficacia y causalidad, tomando como referencia diez empresas industriales, han obtenido un crecimiento rápido después de un período de 3 a 5 años. Un objetivo particular de este estudio era determinar si estas empresas crecían por adaptación a la situación, respondiendo a las demandas del mercado con sus recursos (ejecución) o siguiendo planes y procedimientos previamente determinados hacia los objetivos establecidos (causalidad). La efectividad fue originalmente conectada a la creación de nuevas actividades comerciales y un modelo operativo que cubre las etapas del crecimiento de una organización.

Sin embargo, estudios recientes han considerado la efectividad en el contexto de un negocio existente, integrando el crecimiento empresarial al contexto de empresas establecidas y explorando los roles de la ejecución y la causalidad en sus procesos de crecimiento. Los hallazgos indican el uso de ambas lógicas. Tal es el caso de una investigación que concluye como una de las diez compañías estudiadas sigue los principios operativos de causalidad, a la vez que se analizan los microprocesos asociados con un negocio exitoso establecido por dos hermanos jóvenes (16 y 18), basados en los recientes enfoques procesuales del espíritu empresarial asociado con la teoría de la ejecución y el sensemaking en donde "se crearon oportunidades en lugar de descubrirlas, optimizando recursos familiares limitados durante las primeras etapas de la puesta en marcha” (Jones \& Li, 2017).

$\mathrm{Al}$ analizar la naturaleza de la iniciativa empresarial y la toma de decisiones bajo incertidumbre, se comienza con una nueva tipología de incertidumbre que define y delinea diferentes tipos de contextos inciertos, destacando las diferencias en cómo los empresarios toman decisiones dentro de diferentes tipos de incertidumbre. 
A partir de estos elementos, es posible construir nuevas ideas sobre una teoría del proceso emprendedor, en la que se destaca la naturaleza transitoria de incertidumbre cuando los empresarios hacen ciertos juicios y revisan esos juicios. Se pone entonces en controversia como la incertidumbre, las transiciones a lo largo del proceso de juicio, como el proceso de juicio continúan dinámicamente incluso después de que se realiza un juicio, y cómo la naturaleza de la incertidumbre cambia con el tiempo debido a cambios endógenos y exógenos (Packard, Clark \& Klein, 2017).

Por ejemplo, los estados insulares del Pacífico Sur están a la vanguardia de los cambios climáticos que tienen eventos ambientales severos. Estos pequeños países también enfrentan desafíos económicos y sociales que requieren soluciones empresariales, a partir de un modelo que responda a los factores externos y los eventos fortuitos que influyen directamente en el reconocimiento y la explotación de oportunidades sostenibles.

Por lo tanto, al evaluar la eficacia de este modelo en un estudio en profundidad de Women in Business Development Incorporated, una organización no gubernamental que ayuda a mujeres y familias en Samoa para establecer empresas sostenibles, los hallazgos obtenidos hacen una importante contribución a la literatura emergente sobre el espíritu empresarial, la sostenibilidad y la resiliencia en comunidades en riesgo al mostrar como las capacidades organizacionales clave son necesarios para hacer frente a los shocks exógenos en este contexto (Gray, Duncan, Kirkwood, \& Walton, 2014).

El conocimiento de cómo se lleva a cabo el mercadeo emprendedor en el sector industrial es actualmente bastante débil. Según estudios adelantados por Yang \& Gabrielsson (2017) sobre el proceso de toma de decisiones de marketing de los empresarios en nuevas empresas internacionales y que operan en mercados de alta tecnología, los hallazgos destacan que los empresarios se alternan formas de comercialización causales y efectivas como resultado de su emprendimiento ambidiestro ante las variaciones en la incertidumbre interna, la incertidumbre tecnológica y cualquier turbulencia del mercado que llegue a presentarse.

Aspectos como este, dejan entrever la necesidad de analizar el por qué para algunas empresas las fronteras no son limitantes, "como resultado de la combinación, desde el principio, de recursos y capacidades geográficamente dispersos y desafiando la lógica del hogar (Da Rocha, Simões, de Mello \& Carneiro, 2017).

En suma, la teorización constructivista actual depende en gran medida de lógicas causales y, por lo tanto, postula que los empresarios persiguen o abandonan las oportunidades percibidas poco después de su inicio. Sin embargo, los hallazgos dan cuenta de "procesos menos directos involucrados en el establecimiento de emprendimientos empresariales" (Metzger \& King, 2015), de tal forma que la generación de investigaciones de este tipo, enriquecen la literatura sobre la lógica empresarial de la toma de decisiones, mostrando a los emprendedores nacientes lo indispensable del uso de la efectividad y la causalidad.

En este sentido, estudios como los de Stroe, Parida y Wincent (2018) presentan unos resultados, basados en los datos recopilados de 50 emprendedores nacientes, en los que demuestran cómo más que la pasión, la autoeficacia empresarial y la percepción 
del riesgo solo, es su combinación lo que lleva al uso de una lógica causal y efectiva. Se encuentra otro artículo que, investiga dos brechas de investigación importantes en los negocios internacionales cómo los empresarios evalúan las oportunidades empresariales internacionales y el papel del tiempo en el proceso de evaluación, esto basándose en la literatura en los modelos de toma de decisiones y la base filosófica de la oportunidad, este estudio emplea la metodología y el análisis de contenido de Gioia para examinar cómo los fundadores de 15 firmas de internacionalización temprana (Chandra, 2017).

Por último, se hace importante destaca que, a eficacia se propuso por primera vez como el marco de toma de decisiones de un experto emprendedor frente a la incertidumbre, pero las aplicaciones de la efectividad más allá de la condición de incertidumbre se han visto llamado menos la atención. No obstante, usando un modelo de simulación basado en agentes, en los que se investiga la efectividad de efectuación relativa a la causalidad en contextos inciertos y riesgosos, la simulación supera a las deficiencias de los protocolos de pensar en voz alta normalmente utilizados en la investigación de la ejecución, por lo tanto, se sugiere que "esa efectividad supera a la causalidad en contextos tanto arriesgados como inciertos hasta que el emprendedor puede predecir el futuro correctamente (Welter \& Kim, 2018).

\section{CONCLUSIÓNes}

A partir de la revisión bibliográfica del tema "modelo efectual", el análisis de resultados presentado en forma de árbol (Tree of Sciencie) para entender, de forma visual, la evolución de este tema, se concluyó que los artículos ubicados en la "raíz" fueron catalogados como la base de la teoría, los ubicados en el "tronco" fueron los que le dieron estructura al tema del modelo efectual y los situados en las "ramas" se definieron como las diferentes perspectivas. Como resultado final, se muestra la importancia del modelo efectual como "una oportunidad para los emprendedores en la toma de decisiones aplicando dichos principios" (Ahi, Baronchelli, Kuivalainen, \& Piantoni, 2017).

A su vez, se destaca que, a pesar de la creciente investigación en emprendimiento internacional muy poco se conoce sobre los procesos de cómo los empresarios reconocen y buscan oportunidades en los mercados internacionales. Para proporcionar una respuesta a esta "brecha en el proceso de oportunidad", se basó en el marco de conjuntos de ideas de la literatura de emprendimiento. La investigación demuestra la efectividad del nuevo enfoque del modelo efectual como lente y marco metodológico para avanzar en la investigación relacionada con el emprendimiento.

Estos resultados preliminares revelan un nuevo concepto denominado modelo efectual que, implica el descubrimiento, creación, creación de prototipos y ampliación de oportunidades, que sustenta "la creación de nuevas oportunidades utilizando los recursos disponibles con los que cuenta el emprendedor principiante" (Chandra, Styles \& Wilkinson, 2015). La identidad social del fundador del negocio es crucial para explicar su comportamiento y actitud en el negocio, tomando decisiones con base en cómo las identidades sociales influyen en la forma de gestión del emprendedor, lo que 
lleva a concluir que "la efectividad canaliza los efectos de identidades específicas que permiten al emprendedor lograr resultados provechosos" (Estrada, Verdú \& Gómez, 2018).

Siendo el propósito del presente artículo, el identificar los principales aportes en el modelo efectual, recurriendo a la herramienta Web ToS. ToS, de forma efectiva se muestran los resultados en forma de árbol y de esta manera se realiza una descripción cronológica, a partir de la cual es posible concluir como, el modelo efectual es un campo de estudio valioso compuesto por un marco teórico, que incorpora la información obtenida de múltiples aspectos que se han explorado con la idea de vincular a académicos de otras disciplinas a integrarse con el propósito de generar un conjunto de información sistemática sobre el emprendimiento.

Algunas limitaciones de la investigación fueron la utilización de sólo una base de datos para la selección de artículos científicos, pues se encontraron artículos en las hojas desconectados con el principal tema de investigación. Para estudios posteriores, se podría realizar el estudio con otras bases de datos como scopus o se podrían identificar las diferentes perspectivas por medio de métodos cualitativos con casos de estudio para validar los hallazgos de la presente investigación.

Respecto a la lógica de la toma de decisiones utilizadas por las nuevas empresas para desarrollar sus modelos de negocio, temática que también fue abordada en esta investigación, y que se centra en las lógicas de efectividad y causalidad y cómo su dinámica da forma al desarrollo de modelos de negocio a lo largo del tiempo, se encontró que la lógica de toma de decisiones efectiva se utilizó predominantemente para generar una propuesta de valor viable para un segmento específico de clientes.

La lógica causal se usa predominantemente para definir otros componentes del modelo de negocio en relación con la propuesta de valor y el segmento de clientes, y cuando surge una escasez de recursos, la lógica causal se reemplaza por un aumento en la eficacia, por lo que se concluye "antes de invertir recursos significativos en un modelo de negocio, fue crucial para que las empresas reduzcan, en la medida de lo posible, la tecnología y el mercado" (Reymen, Berends, Oudehand \& Stultiëns, 2017).

\section{Agradecimientos y Reconomientos}

El presente artículo forma parte de la producción intelectual del Grupo de Investigación Finanzas y Marketing de la Facultad de Administración de la Universidad Nacional de Colombia.

\section{REFERENCIAS}

Ahi, A., Baronchelli, G., Kuivalainen, O. \& Piantoni, M. (2017). International Market Entry: How Do Small and Medium-Sized Enterprises Make Decisions? . Journal of International Marketing, 25(1), 1-21. https://doi.org/10.1509/jim.15.0130

Alvárez, S. A. \& Barney, J. B. (2007). Discovery and creation: alternative theories of entrepreneurial action. Strategic Entrepreneurship Journal, 1(1-2), 11-26. https:// doi.org/10.1002/sej.4 
Arend, R. J., Sarooghi, H. \& Burkemper, A. (2015). Effectuation As Ineffectual? Applying the $3 \mathrm{E}$ Theory-Assessment Framework to a Proposed New Theory of Entrepreneurship. AMOR, 40(4), 630-651. https://doi.org/10.5465/amr.2014.0455

Aria, M. \& Cuccurllo, C. (2017). Bibliometrix: An R-tool for comprehensive science mapping analysis. Journal of Informetrics, 11(4), 959-975. https://doi.org/10.1016/j. joi.2017.08.007

Baker, T. \& Nelson, R. E. (2005). Creating Something from Nothing: Resource Construction through Entrepreneurial Bricolage. Administrative Science Quarterly, 50(3), 329-366. https://doi.org/10.2189/asqu.2005.50.3.329

Brettel, M., Mauer, R., Engelen, A. \& Küpper, D. (2012). Corporate effectuation: Entrepreneurial action and its impact on R\&D project performance. Journal of Business Venturing, 27(2), 167-184. https://doi.org/10.1016/j.jbusvent.2011.01.001

Chandler, G. N., DeTienne, D. R., McKelvie, A. \& Mumford, T. V. (2011). Causation and effectuation processes: A validation study. Journal of Business Venturing, 26(3), 375-390. https://doi.org/10.1016/j.jbusvent.2009.10.006

Chandra, Y. (2017). A time-based process model of international entrepreneurial opportunity evaluation. Journal of International Business Studies, 48(4), 423-451. https://doi.org/10.1057/s41267-017-0068-x

Chandra, Y., Styles, C. \& Wilkinson, I. F. (2015). Opportunity portfolio: Moving beyond single opportunity explanations in international entrepreneurship research. Asia Pacific Journal of Management, 32(1), 199-228. https://doi.org/10.1007/s10490014-9400-1

Da Rocha, A., Simões, V. C., de Mello, R. C. \& Carneiro, J. (2017). From global start-ups to the borderless firm: Why and how to build a worldwide value system. Journal of International Entrepreneurship, 15(2), 121-144. https://doi.org/10.1007/s10843017-0200-9

Dew, N., Read, S., Sarasvathy, S. D. \& Wiltbank, R. (2009). Effectual versus predictive logics in entrepreneurial decision-making: Differences between experts and novices. Journal of Business Venturing, 24(4), 287-309. https://doi.org/10.1016/j. jbusvent.2008.02.002

Estrada, M. E., Verdú, A. J. \& Gómez, J. M. (2018). Influence of the entrepreneur's social identity on business performance through effectuation. European Research on Management and Business Economics, 24(2), 90-96. https://doi.org/10.1016/j. iedeen.2017.11.003

Futterer, F., Schmidt, J. \& Heidenreich, S. (2018). Effectuation or causation as the key to corporate venture success? Investigating effects of entrepreneurial behaviors on business model innovation and venture performance. Long Range Planning, 51(1), 64-81. https://doi.org/10.1016/j.lrp.2017.06.008

Gray, B. J., Duncan, S., Kirkwood, J. \& Walton, S. (2014). Encouraging sustainable entrepreneurship in climate-threatened communities: a Samoan case study. Entrepreneurship and Regional Development, 26(5-6), 401-430. https://doi.org/10.1080/ 08985626.2014 .922622

Jones, O. \& Li, H. (2017). Effectual entrepreneuring: sensemaking in a family-based start-up. Entrepreneurship and Regional Development, 29(5-6), 467-499. https:// doi.org/10.1080/08985626.2017.1297854 
Kalinic, I., Sarasvathy, S. D. \& Forza, C. (2014). "Expect the unexpected": Implications of effectual logic on the internationalization process. International Business Review, 23(3), 635-647. https://doi.org/10.1016/j.ibusrev.2013.11.004

Matalamäki, M. J. (2017). Effectuation, an emerging theory of entrepreneurship - towards a mature stage of the development. Journal of Small Business and Enterprise Development, 24(4), 928-949. https://doi.org/10.1108/JSBED-02-2017-0030

Matalamäki, M., Vuorinen, T., Varamäki, E. \& Sorama, K. (2017). Business Growth in Established Companies; Roles of Effectuation and Causation. Journal of Enterprising Culture, 25(2), 123-148. https://doi.org/10.1142/S0218495817500054

Mauer, R., Wuebker, R., Schlüter, J. \& Brettel, M. (2018). Prediction and control: An agent-based simulation of search processes in the entrepreneurial problem space. Strategic Entrepreneurship Journal, 12(2), 237-260. https://doi.org/10.1002/sej.1271

Metzger, M. L. \& King, J. S. (2015). Extending Constructivist Perspectives on Opportunity Production Through An Incorporation of Effectual Logics. Entrepreneurship Research Journal, 5(4), 323-345. https://doi.org/10.1515/erj-2014-0025

Packard, M. D., Clark, B. B. \& Klein, P. G. (2017). Uncertainty Types and Transitions in the Entrepreneurial Process. Organization Science, 28(5), 840-856. https://doi. org/10.1287/orsc.2017.1143

Perry, J. T., Chandler, G. N. \& Markova, G. (2012). Entrepreneurial Effectuation: A Review and Suggestions for Future Research. Entrepreneurship Theory and Practice, 36(4), 837-861. https://doi.org/10.1111/j.1540-6520.2010.00435.x

Read, S., Song, M. \& Smit, W. (2009). A meta-analytic review of effectuation and venture performance. Journal of Business Venturing, 24(6), 573-587. https://doi. org/10.1016/j.jbusvent.2008.02.005

Reymen, I., Berends, H., Oudehand, R. \& Stultiëns, R. (2017). Decision making for business model development: a process study of effectuation and causation in new technology-based ventures. R\&D Management, 47(4), 595-606. https://doi. org/10.1111/radm.12249

Robledo, S., Osorio, G. y López, C. (2014). Networking en pequeña empresa: una revisión bibliográfica utilizando la teoria de grafos. Revista vínculos, 11(2), 6-16. https://doi.org/10.14483/2322939X.9664

Rodríguez-Sepulveda, L. y Orrego-Alzate, C. (2016). Aplicaciones de mezclas de biopolímeros y polímeros sintéticos: Revisión bibliográfica. Revista científica, 25(2), 252-264. https://doi.org/10.14483//udistrital.jour.RC.2016.25.a9

Sarasvathy, S. D. (2009). . Effectuation: Elements of entrepreneurial expertise.Charlottesville, VA: Edward Elgar Publishing. https://doi.org/10.4337/9781848440197

Sarasvathy, S. D. (2001). Causation and Effectuation: Toward a Theoretical Shift from Economic Inevitability to Entrepreneurial Contingency. Academy of Management Review, 26(2), 243-263. https://doi.org/10.2307/259121

Sarasvathy, S. D. \& Dew, N. (2005a). New market creation through transformation. Journal of Evolutionary Economics, 15(5), 533-565. https://oi.org/10.1007/s00191005-0264-x

Sarasvathy, S. \& Dew, N. (2005b). Entrepreneurial logics for a technology of foolishness. Scandinavian Journal of Management, 21(4), 385-406. https://doi.org/10.1016/j. scaman.2005.09.009 
Sarasvathy, S., Kumar, K., York, J. G. \& Bhagavatula, S. (2014). An Effectual Approach to International Entrepreneurship: Overlaps, Challenges, and Provocative Possibilities. Entrepreneurship Theory and Practice, 38(1), 71-93. https://doi. org/10.1111/etap.12088

Shane, S. \& Venkataraman, S. (2000). The Promise of Entrepreneurship as a Field of Research. AMOR, 25(1), 217-226. https://doi.org/10.2307/259271

Stroe, S., Parida, V. \& Wincent, J. (2018). Effectuation or causation: An fsQCA analysis of entrepreneurial passion, risk perception, and self-efficacy. Journal of Business Research, 89, 265-272. https://doi.org/10.1016/j.jbusres.2018.01.035

Sunny, S.-J. \& Chandra, Y. (2013). Growing artificial entrepreneurs: Advancing entrepreneurship research using agent-based simulation approach. International Journal of Entrepreneurial Behavior \& Research, 19(2), 210-237. https://doi. org/10.1108/13552551311310383

Toro-Arias, J. y Rodríguez, M. (2017). Formación en Ética en las Organizaciones: Revisión de la Literatura. Información Tecnológica, 28(2), 167-180. http://dx.doi. org/10.4067/S0718-07642017000200018

Welter, C., \& Kim, S. (2018). Effectuation under risk and uncertainty: A simulation model. Journal of Business Venturing, 33(1), 100-116. https://doi.org/10.1016/j. jbusvent.2017.11.005

Wiltbank, R., Dew, N., Read, S. \& Sarasvathy, S. D. (2006). What to do next? The case for non-predictive strategy. Strategic Management Journal, 27(10), 981-998. Available: http://www.jstor.org/stable/20142390

Yang, M. \& Gabrielsson, P. (2017). Entrepreneurial marketing of international hightech business-to-business new ventures: A decision-making process perspective. Industrial Marketing Management, 64,147-160. https://doi.org/10.1016/j.indmarman.2017.01.007

Zuluaga, M., Robledo, S., Osorio-Zuluaga, G. A., Yathe, L., Gonzalez, D. \& Taborda, G. (2016). Metabolomics and pesticides: systematic literature review using graph theory for analysis of references. NOVA, 14(25), 121-138. https://doi. org/10.22490/24629448.1735

\section{BIODATA}

Jersson Casasbuenas Garzón es Administrador de Empresas y Especialista en Gerencia de Mercadeo de la Universidad EAN (Colombia). Especialista en Pedagogía y Magíster en Educación de la Universidad Pedagógica Nacional (Colombia). ORCID: https://orcid.org/0000-0002-3897-4674

Laura Patricia Giraldo Vélez es Economista Empresarial, Especialista en Desarrollo Gerencial y Magister en Administración de Negocios de la Universidad Autónoma de Manizales. ORCID: https://orcid.org/0000-0002-8417-1821 